\title{
Drawing Out Ideas: Computer Models, Artworks and the Generation of Knowledge
}

\author{
Jonathan Michaels \\ University of Sheffield \\ School of Health and Related Research \\ Regent Court, Regent Street \\ Sheffield S1 4DA, UK \\ j.michaels@sheffield.ac.uk
}

\begin{abstract}
In this paper I describe my implementation of Sol LeWitt's Wall Drawing \#123 in an object-oriented programming language ('Processing'). I defined objects, properties, methods and parameters, based upon explicit or implied details in LeWitt's original work. Where additional assumptions or parameters were needed, I sought evidence from other sources, such as contextual information from the writings of the artist, previous implementations of the same artwork and experimentation with iterations of parameter values. I reflect upon insights generated through considering this process in the context of my experience in a different field, specifically the development of evidence-based guidance in healthcare. This project was carried out as a part of my PhD in Fine Art, which investigates the commonalities and differences between knowledge production through software development for digital art and healthcare modelling, and the implications that this may have for understanding the potential value to other disciplines of encounters with artistic processes or products.
\end{abstract}

Sol LeWitt. Wall drawings. Practice-based research. Software programming. Healthcare models.

\section{INTRODUCTION}

My background is in healthcare research, decision science and evidence based practice and, through my $\mathrm{PhD}$ in Fine Art, I am exploring how art practice and practice-based research can generate knowledge. My personal experience has been that involvement in the art-making process or encounters with artworks often leads to reflections that provide insights relevant to separating areas of my personal or professional life.

Since the Artist Placement Group suggested in the 1960 s that artists had a role in 'societal organisation and decision-making processes' (Stevini 2016) there has been an increasing trend for artists to be embedded in academic, business or other working environments. This is often with the expressed aim of encouraging innovation within a multidisciplinary group (Berthoin Antal 2012).

My starting point for this investigation is the proposition that art may have intrinsic value through its ability to stimulate creative thinking, which may lead to insights in other fields, outside the sphere of art. In contrast to Scrivener's view that 'the proper goal of visual arts research is visual art' (Scrivener 2002), I would argue that visual arts provide a legitimate and potentially valuable way of investigating all aspects of the world in which we live and our relationship to it.

In this inquiry, I explore the process of taking an apparently simple idea, the short set of textual instructions that define one of Sol Lewitt's Wall Drawings, and produce a computer software implementation of the artwork. I describe in detail my development of the software, reflecting upon the issues, ambiguities and uncertainties that became apparent. In doing so I identify resonances and insights relating to other aspects of my experience, particularly around decision-making in healthcare. In making this connection I was initially struck by linguistic and metaphorical links, for example: LeWitt's drafters and those who draft guidance, copying lines and following guidelines, and 'drawing on the evidence'.

In my discussion, I focus on two particular areas of resonance, hierarchies of evidence and the handling of uncertainty. I conclude by considering the implications of this process for my understanding of the potential ways in which 
encounters with art in a multi-disciplinary setting may lead to new, shared insights.

\section{BACKGROUND}

George Box famously stated that 'All models are wrong, but some are useful' (Box 1979). Whilst he was writing from the point of view of a statistician, models are ubiquitous and different versions, often with different terminology, occur in every field. Conceptual, economic, physical and computer models, prototypes, simulations and metaphor are all simplified and generalised representations that have the potential to aid our understanding of the world they represent.

There is increasing use of computer modelling in healthcare research for decision-making at a national policy level for appraising new drugs and treatments (NICE 2014b) and developing clinical guidelines (NICE 2014a). The development of decision models of complex problems in healthcare provides a way to deconstruct a question or area of investigation, and thus promotes explicit consideration of alternative sources of data, underlying assumptions and value judgements.

The description by Lehrer of art as exemplarisation (Lehrer 2012) or Noë's description of art as a 'strange tool' that helps to reconfigure our experience (Noë 2015) seem to suggest ways in which art may provide conceptual models that have a wider role in the generation of knowledge.

\begin{abstract}
"When an artist uses a conceptual form of art, it means that all the planning and decisions are made beforehand and the execution is a perfunctory affair. The idea becomes the machine that makes the art." (LeWitt 1967).
\end{abstract}

Whilst some of his wall drawings are encapsulated in a single sentence (LeWitt 1992), his later writings (LeWitt 1994b, 1994a), and the experience of those who have drafted such drawings (LeWitt \& Legg 1978), demonstrate that their implementation is far from a perfunctory affair. In contrast, software implementations seem to fulfil his criterion, in that all the details encapsulated in the idea, every decision, assumption and parameter that is required to implement the work, must be explicitly defined in the code that drives the machine that makes the art.

LeWitt drawings have been the subject of previous explorations through software implementation. In 2004 Casey Reas developed 'Software Structures' a project commissioned for Artport by the Whitney Museum of American Art. He states:
'The catalyst for this project is the work of LeWitt. I had a simple question: "Is the history of conceptual art relevant to the idea of software as art?" I began to answer the question by implementing three of LeWitt's drawings in software.'

He subsequently went on to develop his own sets of instructions, which he used to investigate different components of the implementation of software structures (Reas 2004). In my own investigation, I consider, not only the relationship between the 'models' of conceptual art and software as art, but also the potential that these artistic models might have for providing insights into unrelated fields. I start by describing the process of developing a computer implementation of the specific work, Wall Drawing \#123, followed by reflection on the questions that arose in the process and where I felt that there was potential for knowledge generation.

\section{PROCESS}

Wall Drawing \#123: Copied lines. The first drafter draws a not straight vertical line as long as possible. The second drafter draws a line next to the first one, trying to copy it. The third drafter does the same, as do as many drafters as possible. Then the first drafter, followed by the others, copies the last line drawn until both ends of the wall are reached. (LeWitt 1972).

I developed a computer-based implementation of the wall drawing using the open-source objectoriented programming (OOP) language 'Processing' (see: https://processing.org) (Reas \& Fry 2014). OOP is a programming paradigm in which data structures are defined as objects, which are members of classes with specified attributes or properties. Objects may be altered by, or interact with each other through a set of defined methods. Whilst there is debate in the computing world about the advantages of OOP (Cardelli 1996), it provides a relatively transparent way to implement a computer model and requires a systematic approach to identifying the objects, properties and methods.

My process started with a conceptual model that defined the objects, their properties and interactions. Where the necessary information was not explicit in the text of the original artwork I sought other sources of evidence. This included indirect inferences from the original text, the diagram provided by the artist (LeWitt 1972), other published evidence from the artist or those involved with the original work, and examination of other sources or implementations of the work (see Section 7 for further details of these). 


\subsection{The Wall}

Since the text describes a 'wall drawing', my first task was to define the wall. Relevant properties are its size (height and width), colour and texture. In practice a computer implementation is likely to have the wall represented by a screen or projection. For the purpose of this implementation my decision was to allow the attached screen/projector to define the size using the software's inbuilt fullScreen() function along with the system 'width' and 'height' variables (in pixels), analogous to the way that physical implementation would be determined by the dimensions of an available wall. LeWitt wrote that the best surface for a drawing was smooth plaster and: 'The physical properties of the wall: height, length, color, material and architectural conditions and intrusions, are a necessary part of the wall drawings.' (LeWitt 1994b).

Galleries have taken great pains to produce smooth white walls. For example, the documentation of the retrospective at the Pompidou Centre in 2013 states that “...the walls for the pencil drawings were given a thin coat of plaster, sanded, then wiped clean. Two coats of primer were followed by three coats of white paint applied in a specific manner using a roller. The walls were then sanded again to produce a perfectly smooth surface." (Gross 2012). In my implementation, I assumed a plain white background. Projection onto a specific wall could introduce the imperfections described by LeWitt, thus creating the unique version depending upon the context in which it is displayed.

\subsection{Drafters}

I next defined the 'drafters', at least three being mentioned in the instructions. Drafters have many potentially relevant properties that may influence the way in which they draw - 'The draftsman's contributions are unforeseen by the artist, even if he, the artist, is the draftsman.' (LeWitt 1994a). For this exercise, simple parameters representing the pressure applied (strength of the line), the accuracy and the reach (see below) of the drafter were included in the model.

\subsection{Drawing implement (pencil)}

Although the drawing implement is not specified in the instructions, LeWitt suggests elsewhere that hard graphite is the material of choice (LeWitt 1994b). For this implementation, lines were drawn in dark grey to represent the suggested graphite, with the 'pencil' object having colour (grey-scale) and width.

\subsection{Lines}

The instructions generate a series of lines. There are many ways to characterise lines but printed, screened or projected digital images ultimately consist of a series of pixels, with colour (in this case grey-scale) and opacity. I represented the vertical lines by a series of points with vertical $(y)$ and horizontal $(x)$ coordinates, each having colour and opacity. The features of the line are derived from 'drawing' processes that inherit characteristics from the drafters and pencils.

\subsection{Drawing the first line}

\subsection{1 'a not straight line'}

A significant issue in this Wall Drawing is what is meant by a 'not straight line', and how to implement this in computer code. This is a loose definition and, for example, a zigzag or sine wave might be considered to qualify. Examination of previous implementations and LeWitt's own diagram (LeWitt 1972) suggest that this is not what is meant. LeWitt's earliest drawings used mainly straight lines or smooth arcs (LeWitt 1969), but he introduced 'not straight lines' in Wall Drawing \#46, stating that this was in honour of Eva Hesse, who had recently died 'as a way of paying homage to the organic contours that were a hallmark of Hesse's art' (Lippard, Roberts \& Swenson 2014).

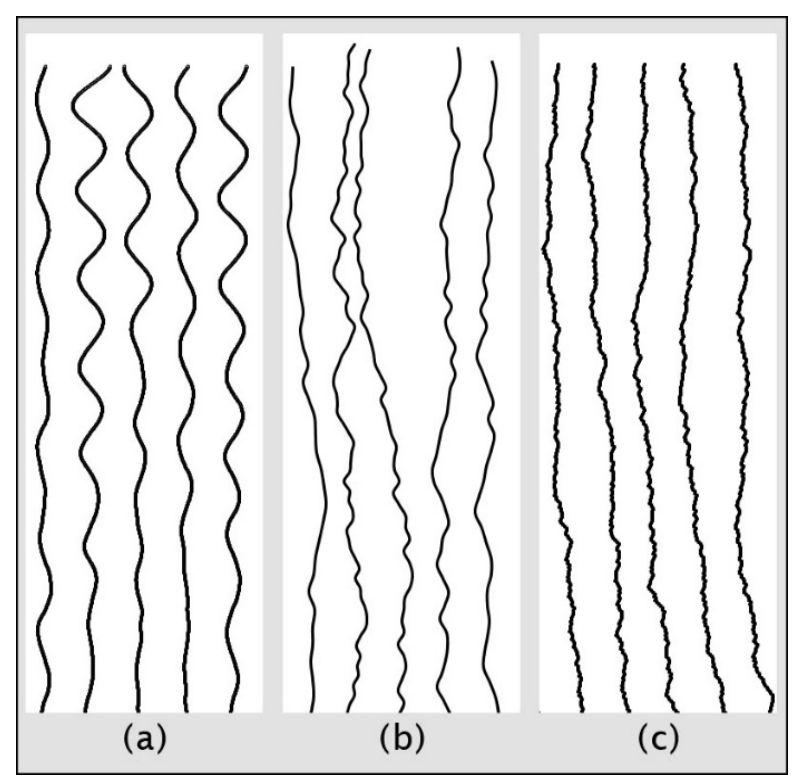

Figure 1: Samples of 'not straight lines' based upon (a) randomly varying lateral velocity parameter, (b) Catmull-Rom splines, and (c) random lateral movements.

Creating 'organic contours' through a computer implementation was done through a process of trial and error. My first attempt used a parameter for lateral velocity, which varied within predetermined bounds (Figure 1a). Further versions used the curve function in Processing, based upon an 
implementation of Catmull-Rom splines (Catmull \& Rom 1974) (Figure $1 \mathrm{~b}$ ) and random lateral movements, within a limited range (Figure 1c). The final version (Figure 2) used the inbuilt noise function within Processing, based upon Perlin noise (Perlin 2004).

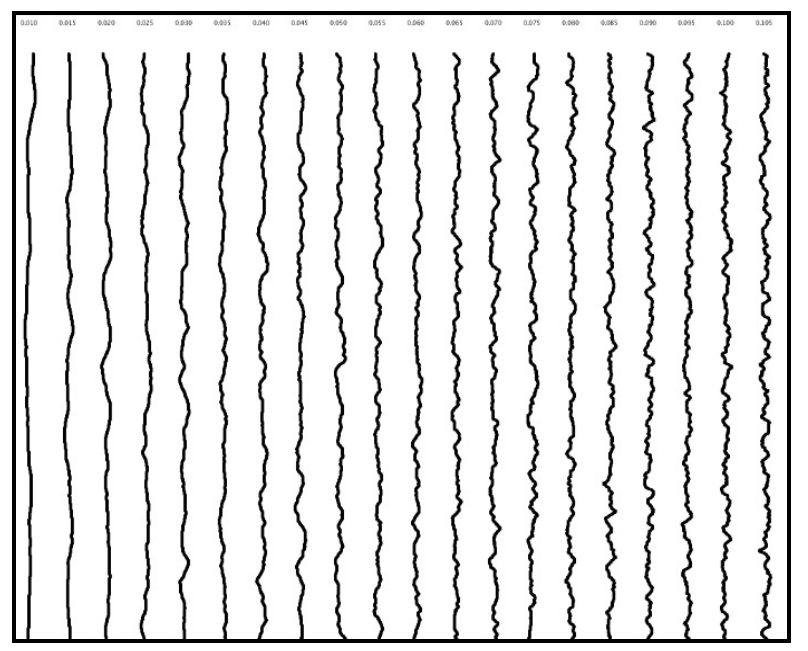

Figure 2: Samples of 'not straight lines' based upon Perlin noise, using different scale parameters.

\subsection{2 'as long as possible'}

The instructions specify that the first line should be 'as long as possible'. For a vertical line on a wall, this would intuitively seem to be from the floor to the ceiling. LeWitt had previously specified this in Wall Drawing \#103, which consists of 'Not straight vertical lines from floor to ceiling...' (Weck \& Morton 2012). He did not specify this and his diagram shows lines of variable length, suggesting that the line length depends upon the reach of the individual drafters. Lynne Cooke, Dia:Beacon curator in 2006, when Drawing \#123 was installed, writes '...differences in lengths of the individual lines reflect different heights and hence different reaches among the ten or so draftspersons involved in its execution...' (Cooke 2009).

\subsubsection{Where to start?}

The starting position on the wall is not specified in the instructions and different implementations suggest different approaches. At Magasin III and Concordia the drawing appears to start at one side of the wall, but 'until both ends of the wall are reached' seems to suggest starting away from an end, and my decision was to take this approach.

\subsubsection{Drafter or pencil?}

LeWitt writes: 'Different draftsman produce lines darker or lighter and closer or farther apart.' (LeWitt 1994b). In this model, I have treated the pencil as contributing a colour and width and the drafter the pressure on the pencil, determining the opacity of the line. This raises a new question - do drafters share a single pencil, have their own pencil, or have a choice of pencils for each new line?
Examination of previous implementations suggests different approaches. At Magasin III all lines appear similar in weight, colour and thickness, at Dia:Beacon they vary in weight and darkness, but not in a regular pattern, whereas at the Addison Gallery there is a regular repeating pattern, suggesting each drafter used a particular pencil.

\subsection{Drawing copied lines}

\subsubsection{Accuracy}

To complete the drawing each drafter, in turn, copies the last line until both ends of the wall are reached. A computer implementation could make exact copies at a fixed distance, but in practice drafters will vary in the closeness and accuracy of the copies. This was simulated by adding some random variation in the distance between lines and additional Perlin noise, based upon a parameter representing the accuracy of the individual drafters.

\subsubsection{Reaching the end}

Since the first drafter draws a line 'as long as possible' based upon their reach, subsequent drafter may have more or less reach. The question arises as to whether copied lines should be limited to the length of the line that is being copied. This is not specified and different approaches have been taken. The Magasin II implementation shows the lines reducing, presumably to the height that can be reach by the shortest drafter and then remaining at this level, whereas other implementations and LeWitt's diagram, have lines of different length, suggesting that drafters extended lines beyond the one they were copying. This is the approach I took.

\subsection{Final implementation}

For the final implementation, each parameter was generated randomly within a given range. There were between 3 and 12 drafters, with their own reach, accuracy and weight used in drawing, and with the colour and width of their pencil. Figure 3 shows one run of the programme, but each would result in a different version of the drawing.

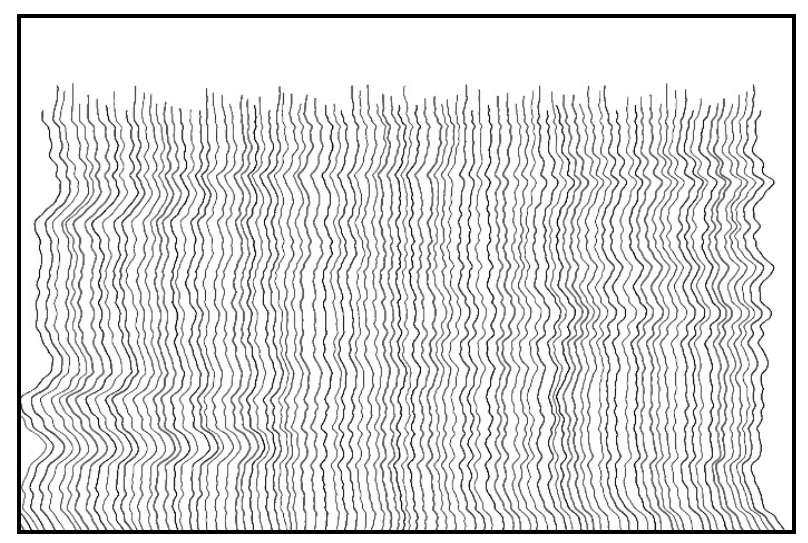

Figure 3: Result of running final version of software implementation of LeWitt Wall Drawing \#123. 


\section{REFLECTION}

The work described in this paper has been a part of my on-going exploration of the ways in which knowledge is generated, understood and valued in different disciplines. My experience of entering an academic art world from that of clinical medicine and healthcare research has been a fascinating, if sometimes very challenging and uncomfortable transition. I have frequently been surprised by the way in which my art practice has led to unanticipated lines of research, reading and thought processes, which seem to provide me with insights relevant to, and sometimes at odds with, other aspects of my experience. My reflections on the potential connections between my experiences in implementing this artwork and issues in healthcare were not an intended direction at the outset, but came about through a combination of factors: my attempts to learn a new programming language (Processing), a visit to Dia:Beacon, where a number of LeWitt Wall Drawings were on show, and resonances to other contexts that arose in dialogue about the process.

Reflecting on my choice of this specific conceptual work as a starting point, I felt attracted by the indeterminacy that it appears to embody. LeWitt's wall drawings are often compared to musical scores 'Like musicians performing a musical score, the drafters interpret, each time slightly differently and in their own way, the geometric formulae set out by LeWitt.' (Gross 2012). Many of his works are quite prescriptive, filling a wall with well-defined lines in exhaustive combinations. I found this particular piece to be more akin to the lead sheets in a jazz fake book, providing a less rigid framework within which the drafters have more room for improvisation and interpretation.

In describing my software implementation of this work, I realise that I have not provided any specific details of the code (which is available at http://www.jonathanmichaels.co.uk/index.php/research/art-

research/lewitt). In keeping with my introductory comments it seems to me that the process of developing the conceptual model upon which the code is based requires the clarification of all the issues where there might be uncertainty. Coding and running the software then becomes the 'perfunctory affair' to which LeWitt referred (LeWitt 1967). This is in keeping with the conclusions of Casey Reas in describing 'Software Structures' that are independent of a specific technology or programming language (Reas 2004).

Whilst the process led to many reflections, for the purposes of this discussion I have focussed on two main areas: the nature of evidence and the handling of uncertainty.

\subsection{Hierarchies of evidence}

Looking back on my implementation I feel some discomfort in that there are a couple of points on which my model does not remain true to the original text. I am interested in exploring why I made these particular decisions. One point relates to the length of the lines. My implementation, as with those of Dia:Beacon and Addison Gallery, produces lines of differing length, determined by the reach of individual drafters. However, the instructions only apply the qualification 'as long as possible' to the first line. My natural inclination in copying a line would be to make it the same length as the line I am copying, which would produce reducing line length, ending with all lines the length of that of the drafter with the shortest reach, as seen in the Magasin III implementation.

My second concern is that the instructions clearly state that the drafters should copy 'the last line drawn until both ends of the wall are reached'. In my implementation, once one end of the wall is reached I have returned to the first line (rather than the line that was drawn last) and started to copy this in the other direction. It is easy enough to correct these issues (Figure 4), but this might be difficult to achieve in practice in a physical implementation, due to the distance from the last line to be copied after reaching one end of the wall, and it creates a discontinuity, which I find uncomfortable and is not seen in LeWitt's diagram or any other implementation of the work.

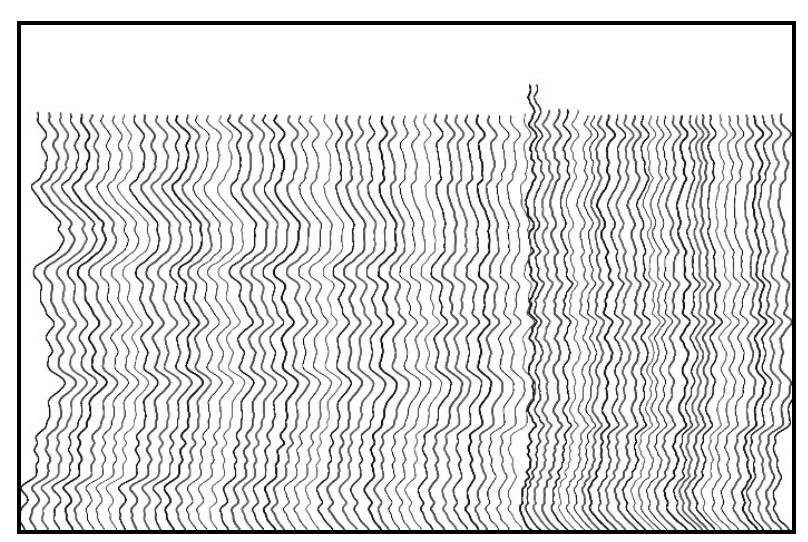

Figure 4: Result of running a modified final version of software implementation of LeWitt Wall Drawing \#123.

I could have resolved these issues by starting one side of the wall, as in the Magasin III version, but chose not to do this, as it would produce a new restriction in that starting the first line close to one end of the wall would create an asymmetrical limit on the 'not-straightness' of the first line.

Reflecting on these issues raised the question of how I had identified evidence and the value 
judgements made where there were alternate, conflicting or missing sources. In my healthcare practice, there is considered to be a "hierarchy of evidence' (Burns, Rohrich \& Chung 2011), often presented as a pyramid, with systematic reviews of randomised controlled trials at the pinnacle (Dartmouth Biomedical Libraries 2017). However, there are concerns that such rigid structures of evidence may obscure underlying value-laden assumptions (Greenhalgh \& Russell 2009), such as those implicit in the choices of outcomes or comparators in the included studies.

I had effectively created my own hierarchy, LeWitt's instructions were my primary source, some 'evidence' being explicitly stated, some implied, or inferred from what is missing (as in assuming that failure to mention extending the lines to the ceiling means that they should be restricted to the reach of the drafter). I also used a variety of other sources, including LeWitt's own writings on wall drawing and evidence from examination other implementations. However, although I thought at time that I was being systematic about use of evidence, my subsequent reflection suggested I used my own judgement (prejudices?) in making some of these decisions. This perhaps illustrates the difficulty of following guidance as an autonomous professional, used to applying one's own judgement and experience.

\subsection{Handling uncertainty}

I encountered various sources of uncertainty in this project and found resonance with the handling of uncertainty in making healthcare decisions. Some uncertainties relate to the extent to which the artwork is open to various interpretations. As LeWitt stated: 'There are decisions which the draftsman makes, within the plan, as part of the plan. Everyone being unique, given the same instructions they would carry them out differently. He would understand them differently.' (LeWitt 1994b). Examples of such uncertainties are those that result from ambiguous or missing aspects of the instructions - what to do when you reach the end of the line or the wall, what drawing implement to use? These are the 'structural uncertainties' seen in healthcare modelling (Bojke et al. 2009), such as treatment options, pathways of care, or more technical decisions, such as the choices of methods used to handle missing data of obtain outcome valuations. My experience in healthcare has been that despite detailed examination of quantitative data, the handling of uncertainty relating to structural assumptions and value judgements is often far less transparent to the nonexpert, and less open to scrutiny.

Another issue is 'parameter uncertainty', the values required for unknown quantitative data, such as the reach of the drafters, the colour and thickness of the pencil. As in healthcare modelling, these can be obtained in different ways - I chose to simulate values within a given range (such as drafters with random reach between $80 \%$ and $90 \%$ of the wall height). I could also have used real-world data by measuring other implementations or seeking published data. Alternatively, the software could model a particular implementation - dimensions and physical parameters taken from a specific wall, a set of intended drafters and drawing implements.

Reflecting on how such sources are used in healthcare modelling brought up some interesting comparisons. Most models developed for policy decisions will use aggregate data with point estimates or distributions representing the average features of the expected population, thus providing average expected outcomes (NICE 2014b). In contrast, there are decision support models that use risk modelling based upon specific characteristics to produce individually tailored estimates of outcome (Middleton, Sittig \& Wright 2016).

One implication of this is that policy decisions, based upon average expectations, may provide different outcomes to individually tailored decisions. Healthcare policy based upon averages may result in denying treatments to some individuals for whom they would be highly cost effective. Whilst tools that allow decisions to be tailored to individuals may improve overall cost effectiveness, there is a tradeoff that demands value judgements. Illness discriminates - many of the parameters that determine outcomes raise ethical issues - age, race, gender, co-existing diseases and the stage of an illness may all determinate of the likely benefit of a treatment. Perhaps this calls for a more fundamental re-examination of the utilitarian approach to maximising health benefit (Cowles et al. 2017, Dolan 2001).

The final source of uncertainty relates to the outcome. Even given clarification about underlying assumptions and details of the drafters and drawing implements, the exact course of the not straight and copied lines is uncertain. This is modelled in my implementation by introducing some randomness into the process but, as seen from the figures, different methods produce characteristic, if unpredictable lines. The choice of the different ways to model uncertainty of outcome is another value judgement, in this case based upon an aesthetic judgement. It is interesting that, although this was not conscious in the process, having drawn links to the 'organic contours' of Eva Hesse's works, I chose to use an algorithm based upon Perlin noise, a method that has been largely used to generated natural looking textures in computer graphics (Lagae et al. 2010). 
This process has parallels in healthcare modelling where value judgements are required in choosing the statistical models used for estimating outcomes such as survival. Whilst attempts have been made to produce guidance for this (Latimer 2011), it is not as simple as identifying a statistical model that best fits the existing data, as this may result in extrapolation of unrealistic survival, so some degree of judgement is often required, which may involve visual inspection (Tremblay, Haines \& Briggs 2015).

\section{CONCLUSIONS}

Just as each implementation of a LeWitt wall drawing will be unique, so the meanings made through encounters with the process or finished result will differ. This paper describes my personal experience of the process of implementing such a drawing and the insights that it generated in relation to my prior experience in healthcare. I have found that the artistic process provides me with a reflective space in which unforeseen associations lead me in unexpected directions and into contact with unfamiliar and sometimes challenging ideas.

Nearly sixty years ago C.P. Snow referred to the dangers inherent in the increasing gulf between science and the humanities (Snow 1959). Since that time the problem has multiplied with many highly specialist fields developing their own language and culture. Crossing the boundaries between disciplines can feel like entering a foreign land, not only with another language, but also with a different value system and understanding of the nature of knowledge. The areas that I have investigated in this paper are not necessarily new to an enlightened art audience, but they are new to me and placing them in a different context feels to have been a useful process. It seems to me to be likely that someone from another discipline, undertaking the same exercise, might find very different resonances.

This research has been undertaken as part of a project considering the potential of art practice to generate knowledge and the value that it might add in a multi-disciplinary setting. My hypothesis, which I hope to go on to examine further, is that the intrinsic value of art lies, at least in part, in its potential to act a model or exemplar for diverse situations that can lead to useful insights.

\section{ACKNOWLEDGEMENTS}

I would like to express my thanks to my wife, Debbie, for the extensive discussions that have helped to shape my thinking, Linda Candy and Ernest Edmonds for their helpful comments on the manuscript and Casey Reas and the Estate of Sol LeWitt for their permission to use relevant material.

\section{PREVIOUS VERSIONS OF DRAWING \#123}

Addison Gallery of American Art, Andover, MA, (1991). See Cooke (2009).

Dia:Beacon, New York, USA. (2006).

http://www.diaart.org/collection/collection/lewitt-sol-walldrawing-123-copied-lines-the-first-drafter-draws-a-not-straightvertical-line-as-long-as-possible-the-second-drafter-draws-aline-next-to-the-first-one-trying-to-copy-it-the-third-drafterdoes-t

Magasin III, Stockholm, Sweden (2009).

https://www.magasin3.com/en/exhibition/sol-lewitt-seven-walldrawings-2/multimedia-14962-img-2/

Concordia, Montreal, Quebec, Canada (2013). http://www.cafad.ca/wp/?p=84

\section{REFERENCES}

Berthoin Antal, A. (2012) 'Artistic intervention residencies and their intermediaries: A comparative analysis', Organizational Aesthetics, 1: 44-67.

Bojke, L., Claxton, K., Sculpher, M., and Palmer, S. (2009) 'Characterizing structural uncertainty in decision analytic models: a review and application of methods', Value in Health, 12: 739-749.

Box, G. E. P. (1979) Robustness in the Strategy of Scientific Model Building (Defense Technical Information Center: Wisconsin Univ-Madison Mathematics Research Center, USA).

Burns, P. B., Rohrich, R. J., and Chung, K. C. (2011) 'The Levels of Evidence and their role in Evidence-Based Medicine', Plast Reconstr Surg, 128: 305-10.

Cardelli, L. (1996) 'Bad engineering properties of object-orient languages', ACM Computing Surveys (CSUR), 28: 150.

Catmull, E. and Rom, R. (1974) 'A class of local interpolating splines', Computer aided geometric design, 74: 317-326.

Cooke, L. (2009) 'Wall drawing 123: Copied Lines.' in Sol LeWitt, Susan Cross and Denise Markonish (eds.), Sol LeWitt: 100 views Yale University Press: New Haven, Conn.

Cowles, E., Marsden, G., Cole, A., and Devlin, N. (2017) 'A Review of NICE Methods and Processes Across Health Technology Assessment Programmes: Why the Differences and What is the 
Impact?', Applied Health Economics and Health Policy: $1-9$.

Dartmouth Biomedical Libraries. (2017) 'EvidenceBased Medicine (EBM) Resources'. http://www.dartmouth.edu/ biomed/resources.htmld/guides/eb m_resources.shtml (retrieved 16 March 2017).

Dolan, P. (2001) 'Utilitarianism and the Measurement and Aggregation of Quality Adjusted Life Years', Health Care Analysis, 9: 6576.

Greenhalgh, T. and Russell, J. (2009) 'Evidencebased policymaking: a critique', Perspect Biol Med, 52: 304-18.

Gross, B. (2012) Sol LeWitt Wall Drawings from 1968 to 2007. Centre Pompidou-Metz, Paris, France

Lagae, A., Lefebvre, S., Cook, R., DeRose, T., Drettakis, G., Ebert, D. S., Lewis, J. P., Perlin, K., and Zwicker, M. (2010) "A survey of procedural noise functions." In Computer Graphics Forum, 29(8), 2579-2600. Wiley Online Library.

Latimer, N. (2011) 'NICE DSU technical support document 14: survival analysis for economic evaluations alongside clinical trials-extrapolation with patient-level data', Sheffield: Report by the Decision Support Unit.

Lehrer, K. (2012) Art, Self and Knowledge (Oxford University Press: New York; Oxford).

LeWitt, S. (1967) 'Paragraphs on Conceptual Art', Artforum, 5: 79-83.

LeWitt, S. (1969) Four basic kinds of straight lines: 1. Vertical. 2. Horizontal. 3. Diagonal I. to r. 4. Diagonal r. to I. and their combinations. (Studio International: London).

LeWitt, S. (1972) 'Wall Drawing \#123, Diagram', Andover, USA.

http://accessaddison.andover.edu/Obj11577 (retrieved 26 February 2017).

LeWitt, S. (1992) Wall drawings 1984-1992 (Kunsthalle Bern).

LeWitt, S. (1994a) 'Doing Wall Drawings (Reprinted from Art Now, 1971, Vol 3 No 2).' in Adachiara Zevi (ed.), Sol LeWitt: Critical Texts Libri de AEIUO.

LeWitt, S. (1994b) 'Wall Drawings (Reprinted from Arts Magazine, 1970, Vol 44 No 6).' In Adachiara Zevi (ed.), Sol LeWitt: Critical Texts Libri de AEIUO.

LeWitt, S. and Legg, A. (1978) Sol Lewitt, Museum of Modern Art, New York (Museum of Modern Art).
Lippard, L. R., Roberts, V., and Swenson, K. (2014) Converging Lines: Eva Hesse and Sol LeWitt. Yale University Press.

Middleton, B., Sittig, D., and Wright, A. (2016) 'Clinical Decision Support: a 25 Year Retrospective and a 25 Year Vision', IMIA Yearbook.

NICE. (2014a) 'Developing NICE guidelines: the manual', NICE, London, UK.

https://www.nice.org.uk/process/pmg20 (retrieved 16 March 2017).

NICE. (2014b) 'Guide to the processes of technology appraisal', NICE, London, UK.

https://www.nice.org.uk/process/pmg19 $\quad$ (retrieved 16 March 2017).

Noë, A. (2015) Strange tools: art and human nature (Hill and Wang, a division of Farrar, Straus and Giroux: New York).

Perlin, K. (2004) 'Implementing improved Perlin noise', GPU Gems, chapter 5, 73-85. AddisonWesley.

Reas, C. (2004) '\{Software\} Structures: A text about Software \& Art', Whitney, USA.

http://artport.whitney.org/commissions/softwarestructures2016/ text.html (retrieved 16 March 2017).

Reas, C. and Fry, B. (2014) Processing: A programming handbook for visual designers and artists. (The MIT Press: Cambridge, Massachusetts).

Scrivener, S. (2002) 'The art object does not embody a form of knowledge', University of Hertfordshire, UK.

https://www.herts.ac.uk/ data/assets/pdf file/0008/12311/WP IAAD vol2 scrivener.pdf (retrieved 16 March 2017).

Snow, C. P. (1959) The Two Cultures and the Scientific Revolution: The Rede Lecture 1959 (Cambridge University Press: Cambridge).

Stevini, B. (2016) 'Artist Placement Group Context is Half the Work. A Partial History of the Artist Placement Group.'

http://en.contextishalfthework.net/about-apg/artist-placementgroup/ (retrieved 16 March 2017).

Tremblay, G., Haines, P., and Briggs, A. (2015) 'A criterion-based approach for the systematic and transparent extrapolation of clinical trial survival data', Journal of Health Economics and Outcomes Research, 2: 147-160.

Weck, Z. D. and Morton, T. (2012) Lines of thought (Parasol Unit Foundation for Contemporary Art: London). 